\title{
Resolution of thrombocytopenia, but not polycythemia after ruxolitinib for polycythemia vera with detectable mutation in the exon 12 of the JAK2 gene
}

\author{
Grzegorz Helbig $^{1} \cdot$ Ryszard Wichary $^{1} \cdot$ Karolina Torba $^{1} \cdot$ Sławomira Kyrcz-Krzemien $^{1}$
}

Received: 22 December 2016/ Accepted: 16 January 2017/Published online: 24 January 2017

(C) The Author(s) 2017. This article is published with open access at Springerlink.com

\section{To the Editor}

Data on safety and efficacy of ruxolitinib treatment in myeloproliferative neoplasms (MPN) with concomitant thrombocytopenia are scarce and limited to patients with myelofibrosis (MF). It was demonstrated that $11-16 \%$ of MF patients had platelet (PLT) count $<50 \times 10^{9} / 1$ at diagnosis and the rate of thrombocytopenia increases with disease progression. Thrombocytopenia in MF may be due to ineffective megakaryocytopoiesis as a result of bone marrow fibrosis or results from platelet sequestration and destruction in enlarged spleen [1]. Immune dysregulation with the production of immune complexes or antiplatelet antibodies may account for low platelet count in single cases [2]. It is noteworthy that thrombocytopenia remains an exceptional finding in polycythemia vera (PV).

Ruxolitinib is the first and only approved JAK inhibitor for the treatment of intermediate- and high-risk MF as well as for PV patients with inadequate response to or are intolerant of hydroxyurea. It should be mentioned that thrombocytopenia was identified as the dose-limiting toxicity for ruxolitinib in MF and was the most common cause of dose modifications. Grade 3/4 thrombocytopenia was rarely seen in PV patients receiving ruxolitinib [3].

There has been a limited experience with ruxolitinib in thrombocytopenic patients with MPN, and all available data were derived from studies in MF. Based on these preliminary results, the treatment with low dose of

Grzegorz Helbig

ghelbig@o2.pl

1 Department of Hematology and Bone Marrow

Transplantation, School of Medicine in Katowice, Medical University of Silesia, Dąbrowski Street 25, 40-032 Katowice, Poland ruxolitinib (10 mg daily) was well tolerated and resulted in spleen reduction with PLT count improvement. To date, only few MF patients with PLT count of $<50 \times 10^{9} / 1$ were successfully treated with ruxolitinib [1, 4].

A 25-year-old female initiated a diagnostic workup due to progressive thrombocytopenia. Seven years earlier, she was diagnosed with polycythemia vera (PV) with detectable H538-K539delinsL mutation in the exon 12 of the JAK2 gene. Since her PV diagnosis she was phlebotomized every other month and receiving hydroxyurea and interferon with no effect. During the follow-up, she developed an isolated mild thrombocytopenia $\left(110 \times 10^{9} /\right.$ 1). She did not present a prior history of immune thrombocytopenia or any other autoimmune disorders.

On her current admission the white blood cell (WBC) count was $28.1 \times 10^{9} / 1$ with the predominance of segmented cells in differential (78\%). No myeloid precursors were found. Red blood cell (RBC) and PLT counts were $5.98 \times 10^{6} / \mu 1$ and $21 \times 10^{9} / 1$, respectively. A massive splenomegaly $(40 \mathrm{~cm})$ was found on ultrasonography. On trephine biopsy her marrow cellularity was $100 \%$. She had reticullin fibrosis of grade I and megakaryocytes were numerous with normal maturation. Blast cells were not seen. Cytogenetics detected normal diploid karyotype. She was found to have antiplatelet antibodies and received steroids with no PLT increase. Then, she was started with ruxolitinib at reduced dose of $15 \mathrm{mg}$ daily due to grade 4 thrombocytopenia. A slow increase in PLT count was observed with concomitant spleen reduction. Five months later, her PLT count was $44 \times 10^{9} / 1$, and ruxolitinib dose was increased to $30 \mathrm{mg}$ daily. At 15 months of ruxolitinib treatment, her PLT count was $93 \times 10^{9} / 1$ with further spleen reduction $(17 \mathrm{~cm}$ on ultrasound). Figure 1. However, her RBC and WBC counts were elevated, and she required regular phlebotomies. A repeated trephine biopsy 
Fig. 1 Graphical representation of platelet count, spleen size, and ruxolitinib daily dose during the disease course

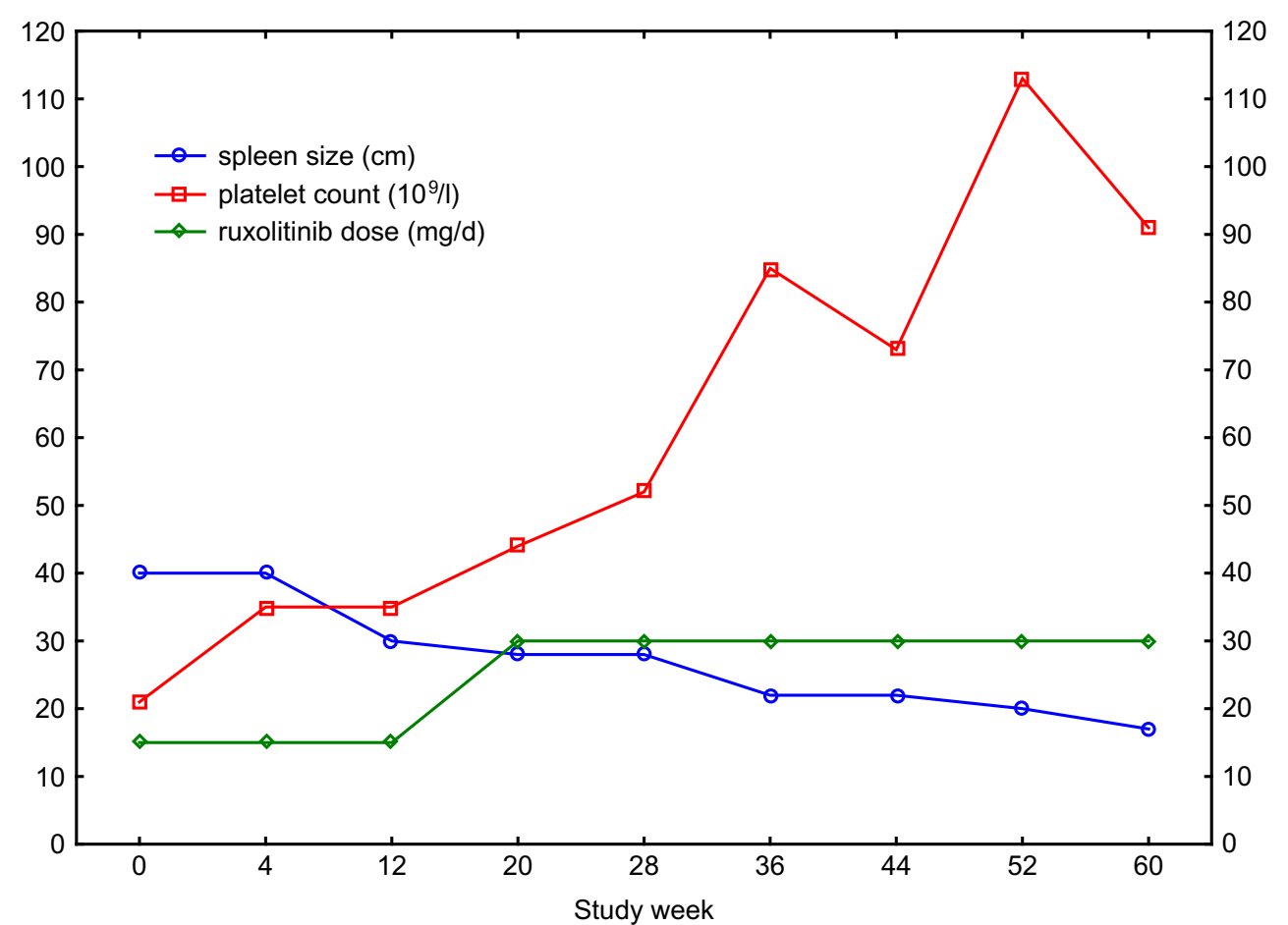

remained unchanged, and the exon 12 mutation was still present.

To date, coexistence of PV with immune thrombocytopenic purpura (ITP) was reported in three cases. All patients were male $>60$ years old with the JAK2 V617Fpositive PV and PLT counts ranging from 8 to $29 \times 10^{9} / 1$. Of note is that they did not exhibit a significant splenomegaly on physical examination, and ITP diagnosis was established by exclusion of other causes of thrombocytopenia. Moreover, low PLT count increased after steroids introduction [2]. Our PV patient presented with severe thrombocytopenia which was related to two independent mechanisms (1) the presence of autoimmunity and (2) hypersplenism. Of note is that steroids were ineffective. The incorporation of ruxolitinib at $15 \mathrm{mg}$ daily resulted in slow platelet recovery without side effects. However no improvements of PV features were observed despite timedependent dose increase and patient remains phlebotomy dependent. It seems likely that the increase in PLT count was most of all associated with reduction of spleen size. It was found that patients with immune thrombocytopenia have an increased Th17 population and defective function of regulatory T cells (Tregs) [5]. The recent data show that treatment with ruxolitinib resulted in a significant reduction of regulatory Tregs and polarization of CD4+ cells from a Th1 to a Th17 phenotype [6]. In this context, ruxolitinib treatment should rather deepen thrombocytopenia than improve it. These data may prove against the beneficial immunomodulatory effect of JAK inhibition in ITP.
To our best knowledge, this is the first report showing the recovery of severe thrombocytopenia after ruxolitinib for PV.

Author's Contributions GH wrote the manuscript and analyzed data; RW, KT, and SKK analyzed data and reviewed the manuscript.

\section{Compliance with ethical standards}

Conflict of interest All authors declare that they have no conflict of interest.

Ethical approval All procedures performed in studies involving human participants were in accordance with the ethical standards of the institutional and/or national research committee and with the 1964 Declaration of Helsinki and its later amendments or comparable ethical standards.

Informed consent Informed consent for publication was obtained from the patient described in the study, and it is available on request.

Open Access This article is distributed under the terms of the Creative Commons Attribution 4.0 International License (http://creativecommons. org/licenses/by/4.0/), which permits unrestricted use, distribution, and reproduction in any medium, provided you give appropriate credit to the original author(s) and the source, provide a link to the Creative Commons license, and indicate if changes were made.

\section{References}

1. Al-Ali HK, Vannucchi AM. Managing patients with myelofibrosis and low platelet count. Ann Hematol. 2016;. doi:10.1007/s00277016-2697-8. 
2. Niscola P, Giovannini M, Scaramucci L, Palombi M, Tendas A, Cupelli L, et al. Idiopathic thrombocytopenic purpura coexisting with polycythemia vera. Asian J Transfus Sci. 2011;5:186-7.

3. Bryan JC, Vestovsek S. Overcoming treatment challenges in myelofibrosis and polycythemia vera: the role of ruxolitinib. Cancer Chemother Pharmacol. 2016;77:1125-42.

4. Bjorn ME, Holmstrom MO, Hasselbalch HC. Ruxolitinib is manageable in patients with myelofibrosis and severe thrombocytopenia: a report on 12 Danish patients. Leuk Lymphoma. 2016;57:125-8.

5. Yazdanbakhsh $\mathrm{K}$, Zhong $\mathrm{H}$, Bao W. Immune dysregulation in immune thrombocytopenia (ITP). Semin Hematol. 2013;50:s63-7.

6. McLornan DP, Khan AA, Harrison CN. Immunological consequences of JAK inhibition: friend or foe. Curr Hematol Malig Rep. 2015;10:370-9. 